\title{
ON THE GROWTH OF THE ONE-DIMENSIONAL REVERSE IMMUNIZATION CONTACT PROCESSES
}

\author{
A. TZIOUFAS, ${ }^{*}$ Heriot-Watt University
}

\begin{abstract}
We are concerned with the variation of the supercritical nearest-neighbours contact process such that first infection occurs at a lower rate; it is known that the process survives with positive probability. Regarding the rightmost infected of the process started from one site infected and conditioned to survive, we specify a sequence of space-time points at which its behaviour regenerates and, thus, obtain the corresponding strong law and central limit theorem. We also extend complete convergence in this case.
\end{abstract}

Keywords: Contact process; Kuczek-type argument

2010 Mathematics Subject Classification: Primary 60K35

Secondary $82 \mathrm{C} 22$

\section{Introduction and main results}

We begin by defining a class of processes that includes the processes we are especially interested in. The nearest-neighbours three-state contact process with parameters $(\lambda, \mu)$ is a continuous-time Markov process $\zeta_{t}$ with state space $\{-1,0,1\}^{\mathbb{Z}}$, elements of which are called configurations and can be thought of as functions from $\mathbb{Z}$ to $\{-1,0,1\}$. The evolution of $\zeta_{t}$ is described locally as follows. Transitions at each site $x, \zeta_{t}(x)$, occur according to the rules

$$
\begin{aligned}
-1 & \rightarrow 1 \\
0 & \rightarrow 1 \text { at rate } \lambda\left|\left\{y=x-1, x+1: \zeta_{t}(y)=1\right\}\right|, \\
1 & \rightarrow 0 \quad \text { at rate } \mu\left|\left\{y=x-1, x+1: \zeta_{t}(y)=1\right\}\right|,
\end{aligned}
$$

for all times $t \geq 0$, where $|B|$ denotes the cardinal of $B \subset \mathbb{Z}$. Typically, the process started from configuration $\eta$ is denoted as $\zeta_{t}^{\eta}$. For general information about interacting particle systems, such as the fact that the above rates specify a well-defined process, we refer the reader to Liggett [11]. We note that the cases $\lambda=\mu$ and $\mu=0$ correspond to the extensively studied processes known as the contact process and the forest fire model, respectively; for an account of various related results and proofs, see Chapters 4, 9, and 10 of Durrett [4] and Part I of Liggett [12]. Furthermore, in the literature various survival aspects of the three-state contact process on the $d$-dimensional lattice were studied by Durrett and Schinazi [7] and Stacey [13], the latter also includes results for the process on homogeneous trees.

The process is thought of according to the following epidemiological interpretation. Given a configuration $\zeta$, each site $x$ is regarded as infected if $\zeta(x)=1$, as susceptible and never infected if $\zeta(x)=-1$, and as susceptible and previously infected if $\zeta(x)=0$. The standard initial configuration is such that the origin is infected while all other sites are susceptible and never infected. We will use $\zeta_{t}^{O}$ to denote the nearest-neighbours three-state contact process started

Received 5 October 2010; revision received 15 March 2011.

* Postal address: Actuarial Mathematics and Statistics, Heriot-Watt University, Edinburgh EH14 4AS, UK.

Email address: a.a.tzioufas@ma.hw.ac.uk 
from the standard initial configuration. We say that the three-state contact process survives if $\mathrm{P}\left(\zeta_{t}^{O}\right.$ survives $)>0$, where the event $\left\{\right.$ for all $t \geq 0$, there exists $\left.x: \zeta_{t}(x)=1\right\}$ is abbreviated as $\left\{\zeta_{t}\right.$ survives $\}$.

For $\zeta_{t}^{O}$, transitions $-1 \rightarrow 1,0 \rightarrow 1$, and $1 \rightarrow 0$ respectively correspond to initial infections, subsequent infections, and recoveries. Accordingly, the initial infection of a site induces a permanent alternation of the parameter proportional to which it will be susceptible; hence, the parameter either decreases, corresponding to (partial) immunization, or increases, i.e. the reverse occurs. Our results concern the three-state contact process under the constraint that $\mu \geq \lambda$ (this explains the title of the paper). When modelling an epidemic, the $\mu \leq \lambda$ case could be a consequence of imperfect inoculation of individuals following their first exposure to the disease, while the $\mu \geq \lambda$ case could be a consequence of debilitation of individuals caused by their first exposure to the disease. Specifically, tuberculosis and bronchitis are plausible examples of a disease that captures the latter characteristic.

When $(\lambda, \mu)$ are such that $\lambda=\mu$, the process is reduced to the well-known contact process. In this case we will identify a configuration with the subset of $\mathbb{Z}$ that corresponds to the set of its infected sites, since states -1 and 0 are effectively equivalent. Also, it is well known that the contact process exhibits a phase transition phenomenon; $\mu_{\mathrm{c}}$ will denote its (one-dimensional nearest-neighbours) critical value, i.e. $0<\mu_{\mathrm{c}}<\infty$, and, if $\mu<\mu_{\mathrm{c}}$, the process dies out, while if $\mu>\mu_{\mathrm{c}}$, the process survives.

It is known that the three-state contact process with parameters $(\lambda, \mu)$ such that $\mu>\mu_{\mathrm{c}}$ and $\lambda>0$ survives; see [7]. We are concerned with the behaviour of the process when survival occurs, assuming additionally that $\mu \geq \lambda$. Theorem 1.1 below summarizes the main results of this paper. In words, parts (i) and (ii) of Theorem 1.1 are respectively a law of large numbers and the corresponding central limit theorem for the rightmost infected, while parts (iii) and (iv) are respectively a law of large numbers and complete convergence for the set of infected sites of the process. In order to demonstrate our results, we introduce some notation. The standard normal distribution function is denoted by $N\left(0, \sigma^{2}\right), \sigma^{2}>0$, and the weak convergence of random variables and of set-valued processes are denoted by $\stackrel{\text { W }}{\rightarrow}$, and by ' $\Rightarrow$ ', respectively. Furthermore, we denote by $\bar{v}_{\mu}$ the upper invariant measure of the contact process with parameter $\mu$, and by $\delta_{\varnothing}$ the probability measure that puts all mass on the empty set. (For general information about the upper invariant measure and weak convergence of set-valued processes, we refer the reader to [12, pp. 34-35].)

Theorem 1.1. Consider $\zeta_{t}^{O}$ with parameters $(\lambda, \mu)$, and let $I_{t}=\left\{x: \zeta_{t}^{O}(x)=1\right\}$ and $r_{t}=$ $\sup I_{t}$. If $(\lambda, \mu)$ are such that $\mu \geq \lambda>0$ and $\mu>\mu_{\mathrm{c}}$, then there exists $\alpha>0$ such that, conditional on $\left\{\zeta_{t}^{O}\right.$ survives $\}$,

(i) $r_{t} / t \rightarrow \alpha$ almost surely;

(ii) $\left(r_{t}-\alpha t\right) / \sqrt{t} \stackrel{\mathrm{W}}{\rightarrow} N\left(0, \sigma^{2}\right)$ for some $\sigma^{2}>0$;

(iii) $\left|I_{t}\right| / t \rightarrow 2 \alpha \theta$ almost surely, where $\theta=\theta(\mu)$ is the density of $\bar{v}_{\mu}$;

(iv) $I_{t} \Rightarrow(1-\beta) \delta_{\varnothing}+\beta \bar{v}_{\mu}$, where $\beta=\mathrm{P}\left(\zeta_{t}^{O}\right.$ survives $)>0$.

We comment on the proof of Theorem 1.1. The cornerstone to proving parts (i) and (ii) is to ascertain the existence of a sequence of space-time points, termed break points, strictly increasing in both space and time, among which the behaviour of $r_{t}$ conditional on $\left\{\zeta_{t}^{O}\right.$ survives $\}$ stochastically replicates; these type of arguments have been established by Kuczek; see [10]. 
We also note that the proofs of parts (iii) and (iv) are based on variations of the arguments for the contact process case due to Durrett and Griffeath; see [2], [6], and [8].

In the next section we introduce the graphical construction, we also present monotonicity and give some elementary coupling results. Section 3 is dedicated to the proof of two exponential estimates that we need in Section 4, where we study break points and provide a proof of Theorem 1.1.

\section{Preliminaries}

\subsection{The graphical construction}

The graphical construction will be used in order to visualize the construction of various processes on the same probability space; we will repeatedly use it throughout this paper.

Consider parameters $(\lambda, \mu)$, and suppose that $\mu \geq \lambda$; the other case is similar. To carry out our construction for all sites $x$ and $y=x-1, x+1$, let $\left(T_{n}^{x, y}\right)_{n \geq 1}$ and $\left(U_{n}^{x, y}\right)_{n \geq 1}$ be the event times of Poisson processes at rates $\lambda$ and $\mu-\lambda$, respectively; furthermore, let $\left(S_{n}^{x}\right)_{n \geq 1}$ be the event times of a Poisson process at rate 1. (All Poisson processes introduced are independent.)

Consider the space $\mathbb{Z} \times[0, \infty)$, thought of as giving a time line to each site of $\mathbb{Z}$; the cartesian product is denoted by ' $x$ '. Given a realization of the aforementioned ensemble of Poisson processes, we define the graphical construction and $\zeta_{t}^{[\eta, s]}, t \geq s$, the nearestneighbours three-state contact process with parameters $(\lambda, \mu)$ started from $\eta$ at time $s \geq 0$, i.e. $\zeta_{s}^{[\eta, s]}=\eta$, as follows. From each point $x \times T_{n}^{x, y}$ we place a directed $\lambda$-arrow to $y \times T_{n}^{x, y}$; this indicates that at all times $t=T_{n}^{x, y}, t \geq s$; if $\zeta_{t-}^{[\eta, s]}(x)=1$ and $\zeta_{t-}^{[\eta, s]}(y)=0$ or $\zeta_{t-}^{[\eta, s]}(y)=-1$, then we set $\zeta_{t}^{[\eta, s]}(y)=1$ (where $\zeta_{t-}(x)$ denotes the limit of $\zeta_{t-\varepsilon}(x)$ as $\varepsilon \rightarrow 0$ ). From each point $x \times U_{n}^{x, y}$ we place a directed $(\mu-\lambda)$-arrow to $y \times U_{n}^{x, y}$; this indicates that any time $t=U_{n}^{x, y}, t \geq s$; if $\zeta_{t-}^{[\eta, s]}(x)=1$ and $\zeta_{t-}^{[\eta, s]}(y)=0$, then we set $\zeta_{t}^{[\eta, s]}(y)=1$. From each point $x \times S_{n}^{x}$ we place a recovery mark; this indicates that at any time $t=S_{n}^{x}, t \geq s$; if $\zeta_{t-}^{[\eta, s]}(x)=1$ then we set $\zeta_{t}^{[\eta, s]}(x)=0$. We introduce the special marks to make a connection with percolation and, hence, the contact process. We define the contact process $\xi_{t}^{A}$ with parameter $\mu$ started from $A \subset \mathbb{Z}$ as follows. We write $A \times 0 \rightarrow B \times t, t \geq 0$, if there exists a connected oriented path from $x \times 0$ to $y \times t$ for some $x \in A$ and $y \in B$ that moves along the arrows (of either type), in the direction of the arrow, and along time lines, in the direction of increasing time, without passing through a recovery mark. Defining $\xi_{t}^{A}:=\{x: A \times 0 \rightarrow x \times t\}, t \geq 0$, we find that $\left(\xi_{t}^{A}\right)$ is a set-valued version of the contact process with parameter $\mu$ started from $A$ infected.

It is important to emphasize that the graphical construction, for fixed $(\lambda, \mu)$, defines all $\zeta_{t}^{[\eta, s]}, t \geq s$, for any configuration $\eta$ and time $s \geq 0$, and all $\xi_{t}^{A}$ for any $A \subset \mathbb{Z}$ simultaneously on the same probability space, i.e. it provides a coupling of all these processes.

Definition 2.1. We shall denote by $\mathcal{L}(\zeta)$ the set of infected sites of any given configuration $\zeta$, i.e. $I(\zeta)=\{y \in \mathbb{Z}: \zeta(y)=1\}$.

To simplify our notation, consistently with Section $1, \zeta_{t}^{[\eta, 0]}$ is denoted as $\zeta_{t}^{\eta}$, and, letting $\eta_{0}$ be the standard initial configuration, $\zeta_{t}^{\left[\eta_{0}, 0\right]}$ is denoted as $\zeta_{t}^{O}$. Additionally, the event $\left\{\mathcal{I}\left(\zeta_{t}^{[\eta, s]}\right) \neq\right.$ $\varnothing$ for all $t \geq s\}$ will be abbreviated below as $\left\{\zeta_{t}^{[\eta, s]}\right.$ survives $\}$.

Finally, we note that we have produced a version of $\zeta_{t}^{\eta}$ via a countable collection of Poisson processes, providing a well-defined process. Indeed, whenever it is assumed that $|\mathcal{I}(\eta)|<\infty$, this is a consequence of standard Markov chains having an almost-surely countable state space; otherwise, this is provided by an argument due to Harris [9] (see also Theorem 2.1 of [5]). 


\subsection{Monotonicity and coupling results}

To introduce monotonicity concepts, we endow the space of configurations $\{-1,0,1\}^{\mathbb{Z}}$ with the componentwise partial order, i.e. for any two configurations $\eta_{1}$ and $\eta_{2}$, we have $\eta_{1} \leq \eta_{2}$ whenever $\eta_{1}(x) \leq \eta_{2}(x)$ for all $x \in \mathbb{Z}$. The following theorem is a known result; for a proof, we refer the reader to Section 5 of [13].

Theorem 2.1. Let $\eta$ and $\eta^{\prime}$ be any two configurations such that $\eta \leq \eta^{\prime}$. Consider the respective three-state contact processes $\zeta_{t}^{\eta}$ and $\zeta_{t}^{\eta^{\prime}}$ with the same parameters $(\lambda, \mu)$ coupled by the graphical construction. For all $(\lambda, \mu)$ such that $\mu \geq \lambda>0, \zeta_{t}^{\eta} \leq \zeta_{t}^{\eta^{\prime}}$ holds. We refer to this property as monotonicity in the initial configuration.

For the remainder of this subsection, we give various coupling results concerning $\zeta_{t}^{O}$, the nearest-neighbours three-state contact process with parameters $(\lambda, \mu)$ started from the standard initial configuration. Let $I_{t}=\mathcal{L}\left(\zeta_{t}^{O}\right), r_{t}=\sup I_{t}$, and $l_{t}=\inf I_{t}$.

We note that both the nearest-neighbours assumption and the assumption that $\mu \geq \lambda$ in all three of the proofs in this subsection are crucial.

The next lemma will be used repeatedly throughout this paper; its proof given below is a simple extension of a well-known result for the nearest-neighbours contact process on $\mathbb{Z}$ (see, e.g. [2]).

Lemma 2.1. Let $\eta^{\prime}$ be any configuration such that $\eta^{\prime}(0)=1$ and $\eta^{\prime}(x)=-1$ for all $x \geq 1$. Consider $\zeta_{t}^{\eta^{\prime}}$ with parameters $(\lambda, \mu)$, and let $r_{t}^{\prime}=\sup \mathcal{I}\left(\zeta_{t}^{\eta^{\prime}}\right)$. For $(\lambda, \mu)$ such that $\mu \geq \bar{\lambda}$, if $\zeta_{t}^{O}$ and $\zeta_{t}^{\eta^{\prime}}$ are coupled by the graphical construction, then the following property holds: for all $t \geq 0$,

$$
r_{t}=r_{t}^{\prime} \quad \text { on }\left\{I_{t} \neq \varnothing\right\}
$$

Proof. We prove the following stronger statement: for all $t \geq 0$,

$$
\zeta_{t}^{O}(x)=\zeta_{t}^{\eta^{\prime}}(x) \text { for all } x \geq l_{t} \text {, on }\left\{I_{t} \neq \varnothing\right\}
$$

Clearly, (2.1) holds for $t=0$. We show that all possible transitions preserve (2.1). An increase of $l_{t}$ (i.e. a recovery mark at $l_{t} \times t$ ), as well as any transition changing the state of any site $x$ such that $x \geq l_{t}+1$, preserves (2.1). It remains to examine transitions that decrease $l_{t}$. By monotonicity in the initial configuration, the possible pairs of $\left(\zeta_{t}^{O}\left(l_{t}-1\right), \zeta_{t}^{\eta^{\prime}}\left(l_{t}-1\right)\right)$ are $(-1,0),(-1,1),(0,0)$, and $(0,1)$. In the $(-1,0)$ case, $(2.1)$ is preserved because $\lambda$-arrows are used for transitions $-1 \rightarrow 1$ as well as $0 \rightarrow 1$, while in the three remaining cases this is obvious. This completes the proof of (2.1).

The next lemma will be used in the proof of the two final parts of Theorem 1.1; its proof is a simple variant of that of Lemma 2.1 and is thus omitted.

Lemma 2.2. Let $\xi_{t}^{\mathbb{Z}}$ be the nearest-neighbours contact process with parameter $\mu$ started from $\mathbb{Z}$. For $(\lambda, \mu)$ such that $\mu \geq \lambda>0$, if $\zeta_{t}^{O}$ and $\xi_{t}^{\mathbb{Z}}$ are coupled by the graphical construction, the following property holds: for all $t \geq 0$,

$$
I_{t}=\xi_{t}^{\mathbb{Z}} \cap\left[l_{t}, r_{t}\right] \text { on }\left\{I_{t} \neq \varnothing\right\} .
$$

Definition 2.2. For all integers $k$, let $\eta_{k}$ be the configuration such that $\eta_{k}(k)=1$ and $\eta_{k}(y)=$ -1 for all $y \neq k$. 
Our final coupling result will be used in the definition of break points in Subsection 4.1. To state the lemma, define the stopping times $\tau_{k}=\inf \left\{t: r_{t}=k\right\}, k \geq 1$, and let $R=\sup _{t \geq 0} r_{t}$.

Lemma 2.3. Let $(\lambda, \mu)$ be such that $\mu \geq \lambda>0$, and consider the graphical construction. Consider also the processes $\zeta_{t}^{\left[\eta_{k}, \tau_{k}\right]}, k \geq 1$, started at times $\tau_{k}$ from $\eta_{k}$ (see Definition 2.2). Then, for all $k=1, \ldots, R$, it holds that

$$
\zeta_{t}^{O} \geq \zeta_{t}^{\left[\eta_{k}, \tau_{k}\right]} \text { for all } t \geq \tau_{k}
$$

Proof. We have $\zeta_{\tau_{k}}^{O}(k)=1$, because $\eta_{k}$ is the least infectious configuration such that $\eta_{k}(k)=1$. We also have $\zeta_{\tau_{k}}^{O} \geq \eta_{k}$ for all $k=1, \ldots, R$. By monotonicity in the initial configuration, the proof is completed.

\section{Exponential estimates}

In this section we establish and prove two exponential estimates for the three-state contact process that will be used in Section 4. The proofs are based on a renormalization result of Durrett and Schinazi [7] that is an extension of the well-known work of Bezuidenhout and Grimmett [1].

Subsequent developments require understanding of oriented site percolation. Consider the set of sites $\mathbb{L}=\left\{(y, n) \in \mathbb{Z}^{2}: n \geq 0\right.$ and $y+n$ is even $\}$. For each site $(y, n) \in \mathbb{L}$, we associate an independent Bernoulli random variable $w(y, n) \in\{0,1\}$ with parameter $p>0$; if $w(y, n)=1$, we say that $(y, n)$ is open. We write $(x, m) \rightarrow(y, n)$ whenever there exists a sequence of open sites $(x, m) \equiv\left(y_{0}, m\right), \ldots,\left(y_{n-m}, n\right) \equiv(y, n)$ such that $\left|y_{i}-y_{i-1}\right|=1$ for all $i=1, \ldots, n-m$. Define $\left(A_{n}\right)_{n \geq 0}$ with parameter $p$ as $A_{n}=\{y:(0,0) \rightarrow(y, n)\}$. We write $\left\{A_{n}\right.$ survives $\}$ as an abbreviation for \{for all $\left.n \geq 1: A_{n} \neq \varnothing\right\}$.

The next proposition is the renormalization result. It is a consequence of Theorem 4.3 of [5], in which the comparison assumptions hold due to Proposition 4.8 of [7]. To state the proposition, given constants $L$ and $T$, we define the set of configurations $Z_{y}=\{\zeta: \mid \mathcal{I}(\zeta) \cap$ $\left.[-L+2 L y, L+2 L y] \mid \geq L^{0.6}\right\}$ for all integers $y$.

Proposition 3.1. Let $\eta$ be any configuration such that $\eta \in Z_{0}$, and consider $\zeta_{t}^{\eta}$ with parameters $(\lambda, \mu)$ such that $\mu>\mu_{\mathrm{c}}$ and $\lambda>0$. For all $p<1$, there exist constants $L$ and $T$ such that $\zeta_{t}^{\eta}$ can be coupled to $A_{n}$ with parameter $p$ such that

$$
y \in A_{n} \Rightarrow \zeta_{n T}^{\eta} \in Z_{y}, \quad(y, n) \in \mathbb{L} .
$$

In particular, the process survives.

The first of the exponential estimates that we need for Section 4 is the following.

Proposition 3.2. Consider $\zeta_{t}^{O}$ with parameters $(\lambda, \mu)$. Let $I_{t}=\mathcal{I}\left(\zeta_{t}^{O}\right), r_{t}=\sup I_{t}$, and $R=\sup _{t \geq 0} r_{t}$, and let $\rho=\inf \left\{t: I_{t}=\varnothing\right\}$. If $(\lambda, \mu)$ are such that $\mu>\mu_{\mathrm{c}}$ and $\mu \geq \lambda>0$, then there exist constants $C$ and $\gamma>0$ such that

$$
\mathrm{P}(R \geq n, \rho<\infty) \leq C \mathrm{e}^{-\gamma n}
$$

for all $n \geq 1$.

Proof. Consider the graphical construction for $(\lambda, \mu)$ as in the statement. Recall the componentwise partial order on the space of configurations and the property of monotonicity in the 
initial configuration that were introduced in Subsection 2.2, and the configurations $\eta_{k}$ given in Definition 2.2. By Proposition 3.1, emulating the proof of Theorem 2.30(a) of [12], we have

$$
\mathrm{P}(t<\rho<\infty) \leq C \mathrm{e}^{-\gamma t}
$$

for all $t \geq 0$. To see that the arguments given in [12] apply in this context, note that, by monotonicity in the initial configuration, for any time $s>0$ and any $x \in I_{s}$, considering the process $\zeta_{t}^{\left[\eta_{x}, s\right]}$, we have $\zeta_{t}^{O} \geq \zeta_{t}^{\left[\eta_{x}, s\right]}$ for all $t \geq s$; hence, the proof in [12] applies for $\delta=\mathrm{P}\left(\zeta_{1}^{O} \in Z_{0}\right)>0$.

To prove (3.1), we note that by set theory we have, for all $n \geq 1$,

$$
\mathrm{P}(R>n, \rho<\infty) \leq \mathrm{P}\left(\frac{n}{\lambda}<\rho<\infty\right)+\mathrm{P}\left(\rho<\frac{n}{\lambda}, R>n\right) .
$$

The first term on the right-hand side decays exponentially in $n$ due to (3.2); thus, it remains to prove that the probability of the event $\left\{\sup _{t<n / \lambda} r_{t}>n\right\}$ decays exponentially in $n$, which is immediate because $\sup _{t \in(0, u]} r_{t}$ is bounded above in distribution by the number of events until time $u$ in a Poisson process at rate $\lambda$ and standard large deviation results for Poisson processes.

The following elementary result for independent site percolation and the subsequent geometrical lemma are needed in the proof of Proposition 3.3 below.

Lemma 3.1. Consider $\left(A_{n}\right)$ with parameter $p$, and define $R_{n}=\sup A_{n}, n \geq 0$. For $p$ sufficiently close to 1 , there are strictly positive and finite constants $a, \gamma$, and $C$ such that

$$
\mathrm{P}\left(R_{n}<\text { an, } A_{n} \text { survives }\right) \leq C \mathrm{e}^{-\gamma n}
$$

for all $n \geq 1$.

Proof. Define $A_{n}^{\prime}=\{y:(x, 0) \rightarrow(y, n)$ for some $x \leq 0\}$, and let $R_{n}^{\prime}=\sup A_{n}^{\prime}, n \geq 1$. Because $R_{n}=R_{n}^{\prime}$ on $\left\{A_{n}\right.$ survives $\}$, it is sufficient to prove that $p$ can be chosen sufficiently close to 1 such that, for some $a>0$, the probability of the event $R_{n}^{\prime}<a$ decays exponentially in $n \geq 0$. Letting $B_{n}^{\prime}$ be independent oriented bond percolation on $\mathbb{L}$ with supercritical parameter $\tilde{p}<1$ started from $\{(x, 0) \in \mathbb{L}: x \leq 0\}$, the result follows from the corresponding large deviations result for $B_{n}^{\prime}$ (see [3, Equation (1), Section 11]), because, for $p=\tilde{p}(2-\tilde{p}), B_{n}^{\prime}$ can be coupled to $A_{n}^{\prime}$ such that $B_{n}^{\prime} \subset A_{n}^{\prime}$ holds (see [12, p. 13]).

Lemma 3.2. Let $b$ and $c$ be strictly positive constants. For any $a<c$, we can choose $a$ sufficiently small $\phi \in(0,1)$ that does not depend on $t \in \mathbb{R} \geq 0$ such that, for all $x \in$ $[-b \phi t, b \phi t]$

$$
[x-c(1-\phi) t, x+c(1-\phi) t] \supseteq[-a t, a t], \quad t \geq 0 .
$$

Proof. Note that it is sufficient to consider $x=b r t$; then, simply choose $\phi$ such that $b t r-c(1-\phi) t<-a t$, i.e. for $\phi<(c-a) /(c+b), \phi>0,(3.3)$ holds.

The other exponential estimate we will need in Section 4 is the following.

Proposition 3.3. Let $\bar{\eta}$ be such that $\bar{\eta}(x)=1$ for all $x \leq 0$, and $\bar{\eta}(x)=-1$ otherwise. Consider $\zeta_{t}^{\bar{\eta}}$ with parameters $(\lambda, \mu)$, and let $\bar{r}_{t}=\sup \mathcal{I}\left(\zeta_{t}^{\bar{\eta}}\right)$. If $(\lambda, \mu)$ are such that $\mu>\mu_{\mathrm{c}}$ and $\mu \geq \lambda>0$, then there exist strictly positive and finite constants $a, \gamma$, and $C$ such that

$$
\mathrm{P}\left(\bar{r}_{t}<a t\right) \leq C \mathrm{e}^{-\gamma t}
$$

for all $t \geq 0$. 
Proof. Consider the graphical construction for $(\lambda, \mu)$ as in the statement. Let $p$ be sufficiently close to 1 so that Lemma 3.1 is satisfied. Recall the configurations $\eta_{x}$ given in Definition 2.2. By the proof of Theorem 2.30(a) of [12]—which applies for the reasons explained in the first paragraph of the proof of Proposition 3.2-we find that the total time $\sigma$ until we get a percolation process $A_{n}$ with parameter $p$ that is coupled to $\zeta_{t}^{\left[\eta_{\bar{r}_{\sigma}}, \sigma\right]}$, as explained in Proposition 3.1 (for $\bar{r}_{\sigma} \times(\sigma+1)$ being thought of as the origin), and conditioned on $\left\{A_{n}\right.$ survives $\}$, is exponentially bounded. From this, because $\bar{r}_{t}$ is bounded above in distribution by a Poisson process, there exists a constant $\tilde{\lambda}$ such that the event $\left\{\bar{r}_{\sigma} \times(\sigma+1) \in\right.$ $[-\tilde{\lambda} t d, \tilde{\lambda} t d] \times(0, t d]\}$ for all $d \in(0,1)$ occurs outside some exponentially small probability in $t$. Finally, on this event, by Lemma 3.1 and the coupling in Lemma 2.1, there exists an $\tilde{a}>0$ such that $\bar{r}_{t} \geq \tilde{a} t-\bar{r}_{\sigma}$, again outside some exponentially small probability in $t$. Choosing $\tilde{\lambda}=b$ and $\tilde{a}=c$ in Lemma 3.2 completes the proof.

\section{Main results}

This section is organized as follows. In Subsection 4.1 we prove Theorem 4.1 stated below; based on this theorem, we prove Theorem 1.1 in Subsection 4.2.

Theorem 4.1. Consider $\zeta_{t}^{O}$ with parameters $(\lambda, \mu)$, and let $r_{t}=\sup \mathcal{I}\left(\zeta_{t}^{O}\right)$. Suppose that $(\lambda, \mu)$ are such that $\mu>\mu_{\mathrm{c}}$ and $\mu \geq \lambda>0$. On $\left\{\zeta_{t}^{O}\right.$ survives $\}$ there exist random (but not stopping) times $\tilde{\tau}_{0}:=0<\tilde{\tau}_{1}<\tilde{\tau}_{2}<\cdots$ such that $\left(r_{\tilde{\tau}_{n}}-r_{\tilde{\tau}_{n-1}}, \tilde{\tau}_{n}-\tilde{\tau}_{n-1}\right)_{n \geq 1}$ are independent and identically distributed (i.i.d.) random vectors, where $r_{\tilde{\tau}_{1}} \geq 1$ and $r_{\tilde{\tau}_{n}}=\sup _{t \leq \tilde{\tau}_{n}} r_{t}$. Furthermore, letting $M_{n}=r_{\tilde{\tau}_{n}}-\inf _{t \in\left[\tilde{\tau}_{n}, \tilde{\tau}_{n+1}\right)} r_{t}, n \geq 0$, we find that $\left(M_{n}\right)_{n \geq 0}$ are i.i.d. random variables, where $M_{n} \geq 0$. Finally, $r_{\tilde{\tau}_{1}}, \tilde{\tau}_{1}$, and $M_{0}$ are exponentially bounded.

\subsection{Break points}

For defining the break points below, consider the graphical construction for $(\lambda, \mu)$ such that $\mu>\mu_{\mathrm{c}}$ and $\mu \geq \lambda>0$. Consider $\zeta_{t}^{O}$, define $r_{t}=\sup \mathcal{I}\left(\zeta_{t}^{O}\right)$, and also define the stopping times $\tau_{k}=\inf \left\{t: r_{t}=k\right\}, k \geq 0$. Let $\eta_{k}$ be as in Definition 2.2. The break points defined below form a unique strictly increasing, in space and in time, subsequence of the space-time points $k \times \tau_{k}, k \geq 1$, such that $\zeta_{t}^{\left[\eta_{k}, \tau_{k}\right]}$ survives. The origin $0 \times 0$ is a break point, i.e. our subsequence is identified on $\left\{\zeta_{t}^{O}\right.$ survives $\}$.

Definition 4.1. Define $\left(K_{0}, \tau_{K_{0}}\right)=(0,0)$. For all $n \geq 0$ and $K_{n}<\infty$, we inductively define

$$
K_{n+1}=\inf \left\{k \geq K_{n}+1: \zeta_{t}^{\left[\eta_{k}, \tau_{k}\right]} \text { survives }\right\},
$$

and $X_{n+1}=K_{n+1}-K_{n}$. Furthermore, we define

$$
\Psi_{n+1}=\tau_{K_{n+1}}-\tau_{K_{n}} \quad \text { and } \quad M_{n}=K_{n}-\inf _{\tau_{K_{n}} \leq t<\tau_{K_{n+1}}} r_{t} .
$$

We refer to the space-time points $K_{n} \times \tau_{K_{n}}, n \geq 0$, as the break points.

Let $\tilde{\tau}_{n}:=\tau_{K_{n}}, n \geq 0$, in the definition of the break points given above. Then, to prove Theorem 4.1, it is sufficient to prove the following two propositions.

Proposition 4.1. $K_{1}, \tau_{K_{1}}$, and $M_{0}$ are exponentially bounded.

Proposition 4.2. $\left(X_{n}, \Psi_{n}, M_{n-1}\right)_{n \geq 1}$ are i.i.d. vectors.

Definition 4.2. Given a configuration $\zeta$ and an integer $y \geq 1$, we define the configuration $\zeta-y$ by $(\zeta-y)(x)=\zeta(y+x)$ for all $x \in \mathbb{Z}$. 
We shall denote by $\mathcal{F}_{t}$ the sigma-algebra associated to the ensemble of Poisson processes used for producing the graphical construction up to time $t$.

The setting of the following lemma is important to what follows.

Lemma 4.1. Let $\bar{\eta}$ be such that $\bar{\eta}(x)=1$ for all $x \leq 0$, and $\bar{\eta}(x)=-1$ otherwise. Consider $\zeta_{t}^{\bar{\eta}}$ with parameters $(\lambda, \mu)$. Define $\bar{r}_{t}=\sup \mathcal{I}\left(\zeta_{t}^{\bar{\eta}}\right)$, and define the stopping times $T_{n}=\inf \left\{t: \bar{r}_{t}=\right.$ $n\}, n \geq 0$. Let $(\lambda, \mu)$ be such that $\mu \geq \lambda>0$ and $\mu>\mu_{\mathrm{c}}$, and consider the graphical construction.

Let $Y_{1}:=1$, and consider $\zeta_{t}^{1}:=\zeta_{t}^{\left[\eta_{Y_{1}}, T_{1}\right]}$. We let $\rho_{1}=\inf \left\{t \geq T_{1}: \mathcal{L}\left(\zeta_{t}^{1}\right)=\varnothing\right\}$. For all $n \geq 1$, proceed inductively. On the event $\left\{\rho_{n}<\infty\right\}$, let

$$
Y_{n+1}=1+\sup _{t \in\left[T_{Y_{n}}, \rho_{n}\right)} \bar{r}_{t},
$$

and consider $\zeta_{t}^{n+1}:=\zeta_{t}^{\left[\eta_{Y_{n+1}}, T_{Y_{n+1}}\right]}$. We let $\rho_{n+1}=\inf \left\{t \geq T_{Y_{n+1}}: \mathcal{I}\left(\zeta_{t}^{n+1}\right)=\varnothing\right\}$. On the event that $\left\{\rho_{n}=\infty\right\}$, let $\rho_{l}=\infty$ for all $l>n$. Define the random variable $N=\inf \{n \geq$ $1: \rho_{n}=\infty$. We have

$$
Y_{N}=\inf \left\{k \geq 1: \zeta_{t}^{\left[\eta_{k}, T_{k}\right]} \text { survives }\right\}
$$

and

$$
\bar{r}_{t}=\sup \mathcal{I}\left(\zeta_{t}^{n}\right) \text { for all } t \in\left[T_{Y_{n}}, \rho_{n}\right) \text { and } n \geq 1
$$

Furthermore,

$$
\left(\zeta_{t+T_{1}}^{1}-1\right)_{t \geq 0} \text { is independent of } \mathcal{F}_{T_{1}} \text { and is equal in distribution to }\left(\zeta_{t}^{O}\right)_{t \geq 0}
$$

and

conditional on $\left\{\rho_{n}<\infty, Y_{n+1}=w\right\}, w \geq 1,\left(\zeta_{t+T_{Y_{n+1}}^{n+1}}-w\right)_{t \geq 0}$ is independent

$$
\text { of } \mathcal{F}_{T_{Y_{n+1}}} \text { and is equal in distribution to }\left(\zeta_{t}^{O}\right)_{t \geq 0} \text {. }
$$

Proof. Equation (4.1) is a consequence of Lemma 2.3. To see this, note that Lemma 2.3 yields, for all $n \geq 1$ on $\left\{\rho_{n}<\infty\right\}, \rho_{n} \geq \inf \left\{t \geq T_{k}: \mathcal{I}\left(\zeta_{t}^{\left[\eta_{k}, T_{k}\right]}\right)=\varnothing\right\}$ for all $k=Y_{n}+$ $1, \ldots, Y_{n+1}-1$. Equation (4.2) is immediate from Lemma 2.1.

Note that from Proposition 3.3 we have $T_{n}<\infty$ for all $n \geq 0$ almost surely (a.s.). Then, (4.3) follows from the strong Markov property at time $T_{1}<\infty$ and translation invariance. Equation (4.4) immediately follows by applying the strong Markov property at time $T_{Y_{n+1}}<\infty$, where $T_{Y_{n+1}}<\infty$, because from Proposition 3.2 we have, conditional on $\rho_{n}<\infty, Y_{n+1}<\infty$ a.s.

The connection between the break points and Lemma 4.1 is given by the following coupling result.

Lemma 4.2. Let $\eta^{\prime}$ be any configuration such that $\eta^{\prime}(0)=1$ and $\eta^{\prime}(x)=-1$ for all $x \geq 1$. Consider $\zeta_{t}^{\eta^{\prime}}$ with parameters $(\lambda, \mu)$, and let $r_{t}^{\prime}=\sup \mathcal{I}\left(\zeta_{t}^{\eta^{\prime}}\right)$ and $\tau_{k}^{\prime}=\inf \left\{t \geq 0: r_{t}^{\prime}=k\right\}$, $k \geq 1$. Define the integers

$$
K^{\prime}=\inf \left\{k \geq 1: \zeta_{t}^{\left[\eta_{k}, \tau_{k}^{\prime}\right]} \text { survives }\right\}
$$

and $M^{\prime}=\inf _{0 \leq t \leq \tau_{K}^{\prime}} r_{t}{ }^{\prime}$. Consider further $\zeta_{t}^{O}$ with parameters $(\lambda, \mu)$. For $(\lambda, \mu)$ such that $\mu \geq \lambda>0$ and $\mu>\mu_{\mathrm{c}}$, if $\zeta_{t}^{O}$ and $\zeta_{t}^{\eta^{\prime}}$ are coupled by the graphical construction, it holds that

$$
\left(K^{\prime}, \tau_{K^{\prime}}^{\prime}, M^{\prime}\right)=\left(K_{1}, \tau_{K_{1}}, M_{0}\right) \text { on }\left\{\zeta_{t}^{O} \text { survives }\right\},
$$

where $K_{1}, \tau_{K_{1}}$, and $M_{0}$ are as in Definition 4.1. 
The proof of Lemma 4.2 is an immediate consequence of Lemma 2.1.

Proof of Proposition 4.1. Consider the setting of Lemma 4.1. By the definition of the break points, Definition 4.1, and Lemma 4.2, on $\left\{\zeta_{t}^{O}\right.$ survives $\}$, we have $K_{1}=Y_{N}, \tau_{K_{1}}=T_{Y_{N}}$, and $M_{0}=\inf _{t \leq T_{Y_{N}}} \bar{r}_{t}$. It is thus sufficient to prove that the random variables $Y_{N}, T_{Y_{N}}$, and $\inf _{t \leq T_{Y_{N}}} \bar{r}_{t}$ are exponentially bounded, merely because an exponentially bounded random variable is again exponentially bounded conditional on any set of positive probability.

We have

$$
Y_{N}=1+\sum_{n=2}^{N}\left(Y_{k}-Y_{k-1}\right) \quad \text { on }\{N \geq 2\}
$$

while $Y_{1}:=1$. Using this and Proposition 3.2, we will prove that $Y_{N}$ is bounded above in distribution by a geometric sum of i.i.d. exponentially bounded random variables and, hence, is itself exponentially bounded.

Let $\rho$ and $R$ be as in Proposition 3.2. We define $p_{R}(w)=\mathrm{P}(R+1=w, \rho<\infty)$ and $\bar{p}_{R}(w)=\mathrm{P}(R+1=w \mid \rho<\infty)$ for all integers $w \geq 1$. Also, define $p=\mathrm{P}(\rho=\infty)>0$ and $q=1-p$, where $p>0$ by Proposition 3.1.

By (4.3) in the statement of Lemma 4.1, we have

$$
\mathrm{P}\left(Y_{2}-Y_{1}=w, \rho_{1}<\infty\right)=p_{R}(w), \quad w \geq 1 ;
$$

similarly, from (4.4) in the same statement, we have, for all $n \geq 1$,

$$
\mathrm{P}\left(\rho_{n+1}=\infty \mid \rho_{n}<\infty, Y_{n+1}=z, \mathcal{F}_{T_{Y_{n+1}}}\right)=p
$$

and

$$
\mathrm{P}\left(Y_{n+1}-Y_{n}=w, \rho_{n}<\infty \mid \rho_{n-1}<\infty, Y_{n}=z, \mathcal{F}_{T_{Y_{n}}}\right)=p_{R}(w)
$$

for all $w, z \geq 1$.

Clearly, $\{N=n\}=\left\{\rho_{k}<\infty\right.$ for all $k=1, \ldots, n-1$ and $\left.\rho_{n}=\infty\right\}, n \geq 2$, and, hence,

$$
\left\{\bigcap_{n=1}^{m}\left\{Y_{n+1}-Y_{n}=w_{n}\right\}, N=m+1\right\}=\left\{\bigcap_{n=1}^{m}\left\{Y_{n+1}-Y_{n}=w_{n}, \rho_{n}<\infty\right\}, \rho_{m+1}=\infty\right\}
$$

for all $m \geq 1$. Using this, from (4.7), $m-1$ applications of (4.8), and (4.6), since $p_{R}(w)=$ $q \bar{p}_{R}(w)$, we have

$$
\mathrm{P}\left(\bigcap_{n=1}^{m}\left\{Y_{n+1}-Y_{n}=w_{n}\right\}, N=m+1\right)=p q^{m} \prod_{n=1}^{m} \bar{p}_{R}\left(w_{n}\right)
$$

for all $m \geq 1$ and $w_{n} \geq 1$. From the last display and (4.5), owing to Proposition 3.2, we find that $Y_{N}$ is exponentially bounded by the following elementary conditioning argument. Let $\left(\tilde{\rho}_{k}, \tilde{R}_{k}\right), k \geq 1$ be independent pairs of random variables, each of which is distributed as $(\rho, R)$, and define the geometric random variable $\tilde{N}:=\inf \left\{n \geq 1: \tilde{\rho_{n}}=\infty\right\}$. Then $Y_{N}$ is equal in distribution to $\sum_{k=0}^{\tilde{N}-1} \tilde{R}_{k}, \tilde{R}_{0}:=1$.

We proceed to prove that $T_{Y_{N}}$ and $\inf _{t \leq T_{Y_{N}}} \bar{r}_{t}$ are exponentially bounded random variables. By (4.1), letting $\bar{x}_{t}=\sup _{s \leq t} \bar{r}_{s}$, we have $\left\{T_{Y_{N}}>t\right\}=\left\{\bar{x}_{t} \leq Y_{N}\right\}$; from this and set theory, we have, for any $a>0$,

$$
\begin{aligned}
\mathrm{P}\left(T_{Y_{N}}>t\right) & =\mathrm{P}\left(\bar{x}_{t} \leq Y_{N}\right) \\
& \leq \mathrm{P}\left(\bar{x}_{t}<a t\right)+\mathrm{P}\left(\bar{x}_{t} \geq a t, \bar{x}_{t} \leq Y_{N}\right) \\
& \leq \mathrm{P}\left(\bar{x}_{t}<a t\right)+\mathrm{P}\left(Y_{N} \geq\lfloor a t\rfloor\right) \quad \text { for all } t \geq 0
\end{aligned}
$$


where $\lfloor\cdot\rfloor$ is the floor function. Choosing $a>0$ as in Proposition 3.3, because $\bar{x}_{t} \geq \bar{r}_{t}$, and since $Y_{N}$ is exponentially bounded, we deduce from (4.9) that $T_{Y_{N}}$ is exponentially bounded as well.

Finally, we prove that $M:=\inf _{t \leq T_{Y_{N}}} \bar{r}_{t}$ is exponentially bounded. From set theory,

$$
\mathrm{P}(M<-x) \leq \mathrm{P}\left(T_{Y_{N}} \geq \frac{x}{\mu}\right)+\mathrm{P}\left(T_{Y_{N}}<\frac{x}{\mu},\left\{\bar{r}_{s} \leq-x \text { for some } s \leq T_{Y_{N}}\right\}\right),
$$

because $T_{Y_{N}}$ is exponentially bounded. It is sufficient to prove that the second term on the right-hand side decays exponentially. However, recall that $\bar{r}_{T_{Y_{N}}} \geq 1$; hence,

$$
\begin{aligned}
& \mathrm{P}\left(T_{Y_{N}}<\frac{x}{\mu},\left\{\bar{r}_{s} \leq-x \text { for some } s \leq T_{Y_{N}}\right\}\right) \\
& \quad \leq \mathrm{P}\left(\left(\bar{r}_{t}-\bar{r}_{s}\right)>x \text { for some } s \leq \frac{x}{\mu} \text { and } t \leq \frac{x}{\mu}\right),
\end{aligned}
$$

where the term on the right-hand side decays exponentially in $x$, because $\left(\bar{r}_{t}-\bar{r}_{s}\right), t>s$, is bounded above in distribution by $\Lambda_{\mu}(s, t]$, the number of events of a Poisson process at rate $\mu$ within the time interval $(s, t]$, by use of standard large deviations for Poisson processes, because $\Lambda_{\mu}(s, t] \leq \Lambda_{\mu}(0, x / \mu]$ for any $s, t \in(0, x / \mu]$.

The next lemma is used in the proof of Proposition 4.2.

Lemma 4.3. Consider the setting of the definition of break points, Definition 4.1. For all $n \geq 1$, we have

$$
\left\{\bigcap_{l=1}^{n}\left\{\left(X_{l}, \Psi_{l}, M_{l-1}\right)=\left(x_{l}, t_{l}, m_{l-1}\right)\right\}, \zeta_{t}^{O} \text { survives }\right\}=\left\{\zeta_{t}^{\left[\eta_{z_{n}}, w_{n}\right]} \text { survives, } \tau_{z_{n}}=w_{n}, A\right\}
$$

for some event $A \in \mathcal{F}_{w_{n}}$, where $z_{n}=\sum_{l=1}^{n} x_{l}$ and $w_{n}=\sum_{l=1}^{n} t_{l}$.

Proof. Considering the setting of Lemma 4.1, we trivially have

$$
\left\{\left(Y_{N}, T_{Y_{N}}, \inf _{t \leq T_{Y_{N}}} \bar{r}_{t}\right)=\left(x_{1}, t_{1}, m_{0}\right)\right\}=\left\{\zeta_{t}^{\left[\eta_{x_{1}}, t_{1}\right]} \text { survives, } T_{x_{1}}=t_{1}, B\right\}
$$

for some event $B \in \mathcal{F}_{t_{1}}$; from this and Lemma 4.2, we have

$$
\begin{aligned}
\left\{\left(X_{1}, \Psi_{1}, M_{0}\right)=\left(x_{1}, t_{1}, m_{0}\right), \zeta_{t}^{O} \text { survives }\right\} & =\left\{\zeta_{t}^{\left[\eta_{x_{1}}, t_{1}\right]} \text { survives, } \tau_{x_{1}}=t_{1}, B, \zeta_{t}^{O} \text { survives }\right\} \\
& =\left\{\zeta_{t}^{\left[\eta_{x_{1}}, t_{1}\right]} \text { survives, } \tau_{x_{1}}=t_{1}, B, I_{t_{1}} \neq \varnothing\right\}
\end{aligned}
$$

for all $x_{1} \geq 1, t_{1} \in \mathbb{R}_{+}$, and $m_{0} \geq 0$. As $\left\{I_{t_{1}} \neq \varnothing\right\} \in \mathcal{F}_{t_{1}}$, we have thus proved (4.10) for $n=1$. For general $n \geq 1$, the proof is derived by repeated applications of the above display.

Proof of Proposition 4.2. Consider the setting of the definition of break points, Definition 4.1. Assume that $K_{n}, \tau_{K_{n}}$, and $M_{n-1}$ are a.s. finite. We will prove that

$$
\begin{aligned}
& \mathrm{P}\left(\left(X_{n+1}, \Psi_{n+1}, M_{n}\right)=(x, t, m) \mid \bigcap_{l=1}^{n}\left\{\left(X_{l}, \Psi_{l}, M_{l-1}\right)=\left(x_{l}, t_{l}, m_{l-1}\right)\right\}, \zeta_{t}^{O} \text { survives }\right) \\
& =\mathrm{P}\left(\left(X_{1}, \Psi_{1}, M_{0}\right)=(x, t, m) \mid \zeta_{t}^{O} \text { survives }\right)
\end{aligned}
$$

for all $\left(x_{l}, t_{l}, m_{l-1}\right), x_{l} \geq 1, t_{l} \in \mathbb{R}_{+}, m_{l-1} \geq 0$, and $l=1, \ldots, n$, and, hence, in particular 
that $K_{n+1}, \tau_{K_{n+1}}$, and $M_{n}$ are exponentially bounded. By induction, and since $K_{1}, \tau_{K_{1}}$, and $M_{0}$ are exponentially bounded by Proposition 4.1, (4.11) completes the proof of Proposition 4.2 by Bayes's sequential formula.

It remains however to prove (4.11). Rewrite the conditioning event on the left-hand side of (4.11) according to (4.10) and note that

$$
\left\{\tau_{z_{n}}=w_{n}\right\} \subset\left\{\zeta_{w_{n}}^{O}\left(z_{n}\right)=1 \text { and } \zeta_{w_{n}}^{O}(y)=-1 \text { for all } y \geq z_{n}+1\right\} .
$$

Applying Lemma 4.2 completes the proof by independence of the Poisson processes in disjoint parts of the graphical construction, because $\left(\zeta_{t+w_{n}}^{\left[\eta_{z_{n}}, w_{n}\right]}-z_{n}\right)_{t \geq 0}$ is equal in distribution to $\left(\zeta_{t}^{O}\right)_{t \geq 0}$ by translation invariance.

\subsection{Proof of Theorem 1.1}

We denote by $\overline{\mathrm{P}}$ the probability measure induced by the construction of the process conditional on $\left\{\zeta_{t}^{O}\right.$ survives $\}$, and we denote by $\overline{\mathrm{E}}$ the expectation associated to $\overline{\mathrm{P}}$. Consider the setting of Theorem 4.1, and let $\alpha=\overline{\mathrm{E}}\left(r_{\tilde{\tau}_{1}}\right) / \overline{\mathrm{E}}\left(\tilde{\tau}_{1}\right), \alpha \in(0, \infty)$.

Proof of part (i). Because $r_{\tilde{\tau}_{n}}=\sum_{m=1}^{n}\left(r_{\tilde{\tau}_{m}}-r_{\tilde{\tau}_{m-1}}\right)$ and $\tilde{\tau}_{n}=\sum_{m=1}^{n}\left(\tilde{\tau}_{m}-\tilde{\tau}_{m-1}\right), n \geq 1$, using the strong law of large numbers twice yields

$$
\overline{\mathrm{P}}\left(\lim _{n \rightarrow \infty} \frac{r_{\tilde{\tau}_{n}}}{\tilde{\tau}_{n}}=\alpha\right)=1 .
$$

We prove that indeed $\lim _{t \rightarrow \infty} r_{t} / t=\alpha, \overline{\mathrm{P}}$-a.s. From Theorem 4.1 we have

$$
\frac{r_{\tilde{\tau}_{n}}-M_{n}}{\tilde{\tau}_{n+1}} \leq \frac{r_{t}}{t} \leq \frac{r_{\tilde{\tau}_{n+1}}}{\tilde{\tau}_{n}} \text { for all } t \in\left[\tilde{\tau}_{n}, \tilde{\tau}_{n+1}\right), n \geq 0 .
$$

Furthermore, because $\left(M_{n}\right)_{n \geq 0}, M_{0} \geq 0$, is a sequence of i.i.d. and exponentially bounded random variables, we have

$$
\overline{\mathrm{P}}\left(\lim _{n \rightarrow \infty} \frac{M_{n}}{n}=0\right)=1,
$$

by the first Borel-Cantelli lemma. Consider any $a<\alpha$. By (4.13) we have

$$
\left\{\frac{r_{t_{k}}}{t_{k}}<a \text { for some } t_{k} \uparrow \infty\right\} \subseteq\left\{\limsup _{n \rightarrow \infty}\left\{\frac{r_{\tilde{\tau}_{n}}-M_{n}}{\tilde{\tau}_{n+1}}<a\right\}\right\} ;
$$

however,

$$
\overline{\mathrm{P}}\left(\limsup _{n \rightarrow \infty}\left\{\frac{r_{\tilde{\tau}_{n}}-M_{n}}{\tilde{\tau}_{n+1}}<a\right\}\right)=0 .
$$

To see this, simply use (4.12) and (4.14) to deduce that $\lim _{n \rightarrow \infty}\left(r_{\tilde{\tau}_{n}}-M_{n}\right) / \tilde{\tau}_{n+1}=\alpha, \overline{\mathrm{P}}$-a.s. By use of the upper bound in (4.13) and (4.12), we also have, for any $a>\alpha, \overline{\mathrm{P}}\left(\left\{r_{t_{k}} / t_{k}>\right.\right.$ $a$ for some $\left.\left.t_{k} \uparrow \infty\right\}\right)=0$. This completes the proof of part (i).

Proof of part (ii). We will prove that

$$
\lim _{t \rightarrow \infty} \overline{\mathrm{P}}\left(\frac{r_{t}-\alpha t}{\sqrt{t}} \leq x\right)=\Phi\left(\frac{x}{\sigma^{2}}\right)
$$

for some $\sigma^{2}>0$ and $x \in \mathbb{R}$, where $\Phi$ is the standard normal distribution function, i.e.

$$
\Phi(y):=\frac{1}{\sqrt{2 \pi}} \int_{-\infty}^{y} \exp \left(-\frac{1}{2} z^{2}\right) \mathrm{d} z, \quad y \in \mathbb{R} .
$$


Define $N_{t}=\sup \left\{n: \tilde{\tau}_{n}<t\right\}$; evoking Lemma 2 of [10, pp. 1330-1331], which applies due to Theorem 4.1, we have

$$
\lim _{t \rightarrow \infty} \overline{\mathrm{P}}\left(\frac{r_{N_{t}}-\alpha t}{\sqrt{t}} \leq x\right)=\Phi\left(\frac{x}{\sigma^{2}}\right), \quad x \in \mathbb{R} .
$$

From this, by standard association of convergence concepts, i.e. Slutsky's theorem, it is sufficient to show that

$$
\overline{\mathrm{P}}\left(\lim _{t \rightarrow \infty} \frac{r_{t}-r_{N_{t}}}{\sqrt{t}}=0\right)=1
$$

and that $\sigma^{2}$ is strictly positive. Note however that by Theorem 4.1 we have

$$
\frac{M_{\tilde{N}_{t}}}{\sqrt{t}} \leq \frac{r_{t}-r_{N_{t}}}{\sqrt{t}} \leq \frac{r_{\tilde{\tau}_{N_{t}+1}}-r_{\tilde{\tau}_{N_{t}}}}{\sqrt{t}}
$$

for all $t \geq 0$.

We show that (4.16) follows from (4.17). Because $\left(r_{\tilde{\tau}_{n+1}}-r_{\tilde{\tau}_{n}}\right)_{n \geq 0}, r_{\tilde{\tau}_{1}} \geq 1$, are i.i.d. and exponentially bounded, by the first Borel-Cantelli lemma, and then the strong law of large numbers, we have

$$
\lim _{n \rightarrow \infty} \frac{\left(r_{\tilde{\tau}_{n+1}}-r_{\tilde{\tau}_{n}}\right) / \sqrt{n}}{\sqrt{\tilde{\tau}_{n} / n}}=0 \quad \overline{\mathrm{P}} \text {-a.s. }
$$

From the above display and emulating the argument given in (4.15), we have $\lim _{t \rightarrow \infty}\left(r_{\tilde{\tau}_{N_{t}+1}}-\right.$ $\left.r_{\tilde{\tau}_{N_{t}}}\right) / \sqrt{t}=0, \overline{\mathrm{P}}$-a.s. Similarly, because $\left(M_{n}\right)_{n \geq 0}, M_{0} \geq 0$, are also i.i.d. and exponentially bounded, we also have $\lim _{t \rightarrow \infty} M_{\tilde{N}_{t}} / \sqrt{t}=0, \overline{\mathrm{P}}$-a.s.

Finally, we show that $\sigma^{2}>0$. As in the proof of Corollary 1 of [10], because $\alpha=$ $\overline{\mathrm{E}}\left(r_{\tilde{\tau}_{1}}\right) / \overline{\mathrm{E}}\left(\tilde{\tau}_{1}\right)$, we need to show that $\overline{\mathrm{E}}\left(r_{\tilde{\tau}_{1}} \overline{\mathrm{E}}\left(\tilde{\tau}_{1}\right)-\tilde{\tau}_{1} \overline{\mathrm{E}}\left(r_{\tilde{\tau}_{1}}\right)\right)^{2}>0$. However, because $r_{\tilde{\tau}_{1}} \geq 1$, this follows by Chebyshev's inequality. This completes the proof of part (ii).

For the remainder of the proof, consider the graphical construction for $(\lambda, \mu)$ such that $\mu>\mu_{\mathrm{c}}$ and $\mu \geq \lambda>0$. Consider $\zeta_{t}^{O}$, and let $r_{t}=\sup I_{t}$ and $l_{t}=\inf I_{t}$ be respectively the rightmost and leftmost infected of $I_{t}=\mathcal{I}\left(\zeta_{t}^{O}\right)$. Consider also $\xi_{t}^{\mathbb{Z}}$, the contact process with parameter $\mu$ started from $\mathbb{Z}$. By Lemma 2.2 we have, for all $t \geq 0$,

$$
I_{t}=\xi_{t}^{\mathbb{Z}} \cap\left[l_{t}, r_{t}\right] \quad \text { on }\left\{I_{t} \neq \varnothing\right\} .
$$

Proof of part (iii). Let $\theta=\theta(\mu)>0$ be the density of the upper invariant measure, i.e. $\theta=$ $\lim _{t \rightarrow \infty} \mathrm{P}\left(x \in \xi_{t}^{\mathbb{Z}}\right)$. We prove that $\lim _{t \rightarrow \infty}\left|I_{t}\right| / t=2 \alpha \theta, \overline{\mathrm{P}}$-a.s.

Considering the interval $\left[\max \left\{l_{t},-\alpha t\right\}, \min \left\{r_{t}, \alpha t\right\}\right]$, we have, for all $t \geq 0$,

$$
\left|\sum_{x=l_{t}}^{r_{t}} \mathbf{1}_{\left\{x \in \xi_{t}^{\mathbb{Z}}\right\}}-\sum_{x=-\alpha t}^{\alpha t} \mathbf{1}_{\left\{x \in \xi_{t}^{\mathbb{Z}}\right\}}\right| \leq\left|r_{t}-\alpha t\right|+\left|l_{t}+\alpha t\right| \quad \text { on }\left\{I_{t} \neq \varnothing\right\},
$$

where we denote by $\mathbf{1}_{E}$ the indicator of event $E$. However, by (4.18), we have $\left|I_{t}\right|=$ $\sum_{x=l_{t}}^{r_{t}} \mathbf{1}_{\left\{x \in \xi_{t}\right\}}$ on $\left\{I_{t} \neq \varnothing\right\}$; thus, because $\lim _{t \rightarrow \infty} r_{t} / t=\alpha$ and, by symmetry, $\lim _{t \rightarrow \infty} l_{t} / t=$ $-\alpha, \overline{\mathrm{P}}$-a.s., the proof follows from (4.19) because it is known that, for any $a>0$,

$$
\lim _{t \rightarrow \infty} \frac{1}{t} \sum_{|x| \leq a t} \mathbf{1}_{\left\{x \in \xi_{t}^{\mathbb{Z}}\right\}}=2 a \theta \quad \text { P-a.s. }
$$

(see Equation (19) in the proof of Theorem 9 of [6]). 
Proof of part (iv). Let $\rho=\inf \left\{t \geq 0: I_{t}=\varnothing\right\}$. In the context of set-valued processes, by general considerations (see [5]), it is known that weak convergence is equivalent to convergence of finite-dimensional distributions and that, by inclusion-exclusion, it is equivalent to show that, for any finite set of sites $F \subset \mathbb{Z}$,

$$
\lim _{t \rightarrow \infty} \mathrm{P}\left(I_{t} \cap F=\varnothing\right)=\mathrm{P}(\rho<\infty)+\mathrm{P}(\rho=\infty) \phi_{F}(\varnothing),
$$

where $\phi_{F}(\varnothing):=\lim _{t \rightarrow \infty} \mathrm{P}\left(\xi_{t}^{\mathbb{Z}} \cap F=\varnothing\right)$. By set theory, it is sufficient to prove that $\lim _{t \rightarrow \infty} \mathrm{P}\left(I_{t} \cap F=\varnothing, \rho \geq t\right)=\mathrm{P}(\rho=\infty) \phi_{F}(\varnothing)$, because $\{\rho<t\} \subseteq\left\{I_{t} \cap F=\varnothing\right\}$. However, emulating the proof of the respective result for the contact process (see, e.g. Theorem 5.1 of [8]), we have $\lim _{t \rightarrow \infty} \mathrm{P}\left(\xi_{t}^{\mathbb{Z}} \cap F=\varnothing, \rho \geq t\right)=\mathrm{P}(\rho=\infty) \phi_{F}(\varnothing)$; hence, it is sufficient to prove that

$$
\limsup _{t \rightarrow \infty} \mathrm{P}\left(I_{t} \cap F=\varnothing, \rho \geq t\right) \leq \lim _{t \rightarrow \infty} \mathrm{P}\left(\xi_{t}^{\mathbb{Z}} \cap F=\varnothing, \rho \geq t\right),
$$

because $\left\{I_{t} \cap F=\varnothing, \rho \geq t\right\} \supseteq\left\{\xi_{t}^{\mathbb{Z}} \cap F=\varnothing, \rho \geq t\right\}$ by (4.18).

It remains to prove (4.20). By elementary calculations,

$$
\mathrm{P}\left(I_{t} \cap F=\varnothing, \rho=\infty\right)-\mathrm{P}\left(\xi_{t}^{\mathbb{Z}} \cap F=\varnothing, \rho \geq t\right) \leq \mathrm{P}\left(\xi_{t}^{\mathbb{Z}} \cap F \supsetneq I_{t} \cap F, \rho=\infty\right)
$$

for all $t \geq 0$, where we have used the fact that, by (4.18), $I_{t} \subset \xi_{t}^{\mathbb{Z}}$ for all $t \geq 0$. From the above display and set theory, we have

$$
\begin{aligned}
& \mathrm{P}\left(I_{t} \cap F=\varnothing, \rho \geq t\right)-\mathrm{P}\left(\xi_{t}^{\mathbb{Z}} \cap F=\varnothing, \rho \geq t\right) \\
& \quad \leq \mathrm{P}\left(\xi_{t}^{\mathbb{Z}} \cap F \supsetneq I_{t} \cap F, \rho=\infty\right)+\mathrm{P}(t<\rho<\infty)
\end{aligned}
$$

for all $t \geq 0$; however, the limit as $t \rightarrow \infty$ of both terms on the right-hand side is 0 -for the first term this follows from (4.18), because $\lim _{t \rightarrow \infty} r_{t}=\infty$ and $\lim _{t \rightarrow \infty} l_{t}=\infty, \overline{\mathrm{P}}$-a.s., while, for the second term, this is obvious.

\section{References}

[1] Bezuidenhout, C. E. And Grimmett, G. (1990). The critical contact process dies out. Ann. Prob. 18, 14621482.

[2] Durrett, R. (1980). On the growth of one-dimensional contact processes. Ann. Prob. 8, 890-907.

[3] Durrett, R. (1984). Oriented percolation in two dimensions. Ann. Prob. 12, 999-1040.

[4] Durrett, R. (1988). Lecture Notes on Particle Systems and Percolation. Wadsworth \& Brooks/Cole, Pacific Grove, CA.

[5] Durrett, R. (1995). Ten Lectures on Particle Systems (Lecture Notes Math. 1608). Springer, New York.

[6] Durrett, R. and Griffeath, D. (1983). Supercritical contact processes on $\mathbb{Z}$. Ann. Prob. 11, 1-15.

[7] Durrett, R. Schinazi, R. B. (2000). Boundary modified contact processes. J. Theoret. Prob. 13, 575-594.

[8] Griffeath, D. (1979). Additive and Cancellative Interacting Particle Systems (Lecture Notes Math. 724). Springer, Berlin.

[9] Harris, T. E. (1972). Nearest-neighbor Markov interaction processes on multidimensional lattices. Adv. Math. 9, 66-89.

[10] Kuczek, T. (1989). The central limit theorem for the right edge of supercritical oriented percolation. Ann. Prob. 17, 1322-1332.

[11] Liggett, T. M. (1985). Interacting Particle Systems. Springer, New York.

[12] Liggett, T. M. (1999). Stochastic Interacting Systems: Contact, Voter and Exclusion Processes. Springer, Berlin.

[13] Stacey, A. (2003). Partial immunization processes. Ann. Appl. Prob. 13, 669-690. 